\section{Bone lesions in chronic granulocytic leukaemia}

Sir,

Bone involvement is seen commonly in acute leukaemia but occurs rarely in chronic granulocytic leukaemia (CGL). ${ }^{1-2}$ Among 370 patients with CGL seen between July 1984 to June 1988,14 (3.7\%) developed bone disease. Bone involvement occurred within 1-120 months (median-24 months) of diagnosis and presented with severe localized bone pains $(13 / 14)$, bony swelling $(6 / 14)$, difficulty in walking (4/14) and paraplegia (1/14). The various sites were: spine-4, pelvis-3, skull-3, tibia-4, and femur, mandible, sternum, talus in one patient each. The lesions were single in 10 and multiple in 4 patients. Radiologically the lesions were osteolytic permeative in 10 , multiple punched out in 2 and due to partial collapse of the spine in 4 patients. Peripheral blood and bone marrow examination revealed: chronic phase in 8 patients and accelerated phase and lymphoid blast crisis in 4 and 2 patients respectively. Serum calcium was normal in all patients on at least two repeated occasions. Local radiotherapy in 11 patients resulted in significant relief of symptoms. Three patients were lost to follow-up after the diagnosis of bone lesions.

Three to ten per cent of CGL patients may develop bone involvement during the course of the disease. ${ }^{1-3}$ The most common presenting symptom is localized bone pain followed by bony swelling. Rarely, patients may present with neurological deficits, pathological fracture, or hypercalcaemia. ${ }^{3}$ The ends of long bones, the spine, skull and pelvis are commonly involved. Sternum, mandible and metacarpal may, as seen here, be rarely involved. The lesions may be single, multiple, discrete, punched out or ill-defined. Osteolytic lesions are commonest. Rarely, there may be osteosclerotic or mixed lytic-sclerotic lesions due to myelofibrosis which may complicate the course of CGL. Bone disease due to infection, an unrelated second neoplasm and infarction should also be kept in mind. ${ }^{3}$

Bone lesions are most commonly seen in the late stage of the disease during accelerated phase/blast crisis. Their association with the chronic phase is rare. Interestingly, 8 out of our 14 patients were in the chronic phase with bone involvement. It is speculated that presence of a bone lesion due to leukaemic infiltrates in the chronic phase of CGL may indicate the initial site of clonal proliferation extending later into the remainder of haemopoietic tissue - a situation similar to extramedullary blast crisis described for infiltrates into skin, lymph nodes, breast, meninges etc. ${ }^{1-3}$ This is further supported by the development of a blast crisis in 6 of these patients within 2-12 months. We used hydroxyurea in the chronic phase patients. Whether the use of aggressive chemotherapy in these chronic phase patients could have delayed the onset of blast crisis remains speculative. Here, localized radiotherapy resulted in marked symptomatic relief in all patients, suggesting that this should be used in addition to systemic chemotherapy.

Our experience and other publications ${ }^{1-5}$ suggest that once the bone lesions develop the course is rapidly progressive. The development of blastic transformation results in a rapid clinical deterioration and short survival.

\author{
Lalit Kumar \\ B.L. Baid' \\ Yashwant Singh ${ }^{2}$ \\ V. Kochupillai \\ Departments of Medical Oncology, \\ ${ }^{1}$ Radiotherapy and ${ }^{2}$ Haematology, \\ Institute Rotary Cancer Hospital, \\ All India Institute of Medical Sciences, \\ New Delhi - 110029, India.
}

\section{References}

1. Chabner, B.A., Haskell, C.M. \& Canellos, G.P. Destructive bone lesions in chronic granulocytic leukemia. Medicine 1969, 48: $401-410$.

2. Valimaki, M., Vuopio, P. \& Leiwendahi, K. Bone lesions in chronic myelogenous leukemia. Acta Med Scand 1981, 210: 403-408.

3. Lambertenghi, D.G., Castognone, D., Porro, F. \& Cortelezzi, A. Osteolysis in chronic myeloid leukemia. Tumori 1978, 64: $175-181$.

4. Haskell, C.M. DeVita, V.T. \& Canellos, G.P. Hypercalcemia in chronic granulocytic leukemia. Cancer 1971, 27: 872-880.

5. Campbell, E., Maldonado, W. \& Suharland, G. Painful lytic lesions in an adult with chronic myelogenous leukemia. Cancer 1975, 33: $1354-1356$.

Salmonella enteritidis empyema complicating lupus pleuritis

Sir,

In the absence of concurrent pulmonary infection pleural empyema caused by non-typhoid salmonella is a rare event. In two recent reviews only 16 cases were recorded. ${ }^{1,2}$

Systemic lupus erythematosus (SLE) predisposes to severe salmonella infections, ${ }^{3-5}$ which frequently localize at a site of clinical SLE involvement. ${ }^{3}$ Colonization of sterile serous effusions by salmonella has been reported in other diseases, but not in SLE. ${ }^{6-8}$ We report a patient with long standing lupus pleural effusion who developed bilateral pleural empyema caused by Salmonella enteritidis.

A 26 year old man had SLE diagnosed in 1983. Lupus manifestations had been intestinal vasculitis, arthritis, polyserositis, cutaneous lesions, proteinuria, and interstitial cystitis. Treatment had included prednisone and azathioprine for long periods.

In March 1989 he complained of exertional dyspnoea and a chest X-ray showed bilateral pleural effusion. The pleural fluid was not analysed. The corticosteroid dose was increased, but the effusions persisted. Nine months later, the patient presented with a 5-day history of fever, cough, right pleuritic pain and dyspnoea at rest. A chest 
X-ray showed bilateral pleural effusion. The white blood cell count was $6.6 \times 10^{9} / 1$ with $89 \%$ neutrophils. Thoracocentesis yielded 0.5 litres of a yellow fluid, from which $S$. enteritidis was grown. Blood cultures were sterile. Repeated X-ray films showed no underlying pulmonary involvement. Bilateral thoracostomy tubes were inserted and treatment was ampicillin $\Sigma \mathrm{g} / 4$ hours i.v. for 4 weeks and oral amoxycillin for 2 weeks more, but pleural fluid drainage persisted. A bilateral chemical pleurodesis was performed but a sterile pleural effusion recurred 4 months after finishing antibiotic therapy.

Non-typhoid salmonellae are known to colonize previously damaged tissues and non-lupus pleural and peritoneal effusions have become infected. ${ }^{1,2,6-8}$ Extension from a nearby site, bacteraemic spread from a gastrointestinal source or a dormant focus in the reticuloendothelial system are all pathogenic possibilities. In contrast to other extraintestinal infections, salmonella pleuropulmonary disease usually has an acute onset with symptoms lasting less than a week before a diagnosis is established, ${ }^{2}$ and half the patients have a positive blood or stool culture. ${ }^{2}$

Most other Gram-negative pleuropulmonary infections are nosocomially acquired; salmonella is frequently community acquired. Prior abnormalities of the lungs and pleura are found in about $40 \%$ of patients, malignancy being the most common predisposing condition and $36 \%$ are immunosuppressed, including SLE and corticosteroid-treated individuals. ${ }^{1,2}$

Treatment of salmonella empyema resembles that of other bacterial empyema. Relapse is common and mortality may be as high as $15 \% .^{2}$

Salmonella empyema should be excluded in febrile lupus patients with pleural effusions. Optimal treatment must include drainage and prolonged parenteral effective therapy with a beta-lactam or fluorquinolone antibiotic. Mortality in salmonella pleuropulmonary infections may reach $100 \%$ when antimicrobial agents not active against salmonella are used.'

Alberto Ortiz Diego Giraldez Jesús Egido Manuel Fernández-Guerrero ${ }^{1}$ Departments of Nephrology and 'Infectious Diseases, Fundación Jiménez Diaz, Av Reyes Católicos 2, 28040 Madrid, Spain.

\section{References}

1. Aguado, J.M., Obeso, G., Cabanillas, J.J., FernandezGuerrero, M. \& Alés, J. Pleuropulmonary infections due to nontyphoid strains of salmonella. Arch Intern Med 1990, 150: $54-56$.

2. Cohen, J.I., Bartlett, J.A. \& Corey, G.R. Extraintestinal manifestations of salmonella infections. Medicine 1987, 66: 349-388.

3. Lovy, M.H., Ryan, P.F.J. \& Hughes, G.R.V. Concurrent systemic lupus erythematosus and salmonellosis. $J$ Rheumatol 1981, 8: 605-612.
4. Abramson, S., Kramer, S.B., Radin, A. \& Holzman, R. Salmonella bacteremia in systemic lupus erythematosus. Arthritis Rheum 1985, 28: 75-79.

5. de Luis, C., Pigrau, C., Pahissa, A., Fernandez, F. \& MartinezVazquez, J.M. Infecciones en 96 casos de lupus eritematoso sistemico. Rev Clin Esp 1990, 94: 607-610.

6. Gosset, S., Goursaud, G., Carjuzan, A., Martin, J. Guigui, J. \& Seguin, P. Localisation pleurale d'une salmonella non typhoidique multirésistente. Presse Med 1988, 17: 2200.

7. Reddy, K.R., Chan, J.C., Smiley, D., Jeffers, L.J. \& Schiff, E.R. Spontaneous group B Salmonella enteritidis peritonitis in cirrhotic ascites and AIDS. Am J Gastroenterol 1988, 83: 882-884.

8. Carel, R., Schey, G., Ma'ayan, M. \& Bruderman, I. Salmonella empyema as a complication in malignant pleural effusion. Respiration 1977, 34: 232-235.

\section{Spontaneous oesophageal haematoma presenting as acute myocardial infarction: implications for thrombolytic therapy}

Sir,

Retrosternal chest pain of oesophageal origin is well known to mimic ischaemic chest pain. ${ }^{1}$ The benefits of thrombolytic treatment in acute myocardial infarction are well established and are reported to outweigh the risks. ${ }^{2}$ Speedy thrombolysis has been recommended ${ }^{3}$ and electrocardiographic changes are not considered to be mandatory. ${ }^{4}$ We describe a patient who was a potential candidate for thrombolytic therapy but was subsequently proven to have a spontaneous intramural oesophageal haematoma.

A 66 year old woman with a sudden onset of severe retrosternal chest pain of 1 hour's duration was referred with a suspected myocardial infarction. The pain woke her up from sleep, radiated to her throat and culminated in a sensation of choking. She denied recent vomiting, retching, dysphagia or dyspepsia. Previously she had been fit and well, and was a teetotaller. Clinical examination was normal. The blood count showed a haemoglobin of $13.4 \mathrm{~g} / \mathrm{l}$ and platelet count and white cell count were within normal limits. The electrocardiogram and chest $\mathrm{X}$-ray were normal. Serial cardiac enzymes and coagulation studies were later found to be normal.

Thrombolytic treatment was withheld. The following day she complained of dysphagia and pain on swallowing. Endoscopy showed pronounced bulging of the posterior oesophageal mucosa extending from the upper oesophagus (approximately $25 \mathrm{~cm}$ from the tip of the endoscope) $5 \mathrm{~cm}$ distally. No oesophageal tear, hiatus hernia, gastric or oesophageal lesions were seen. The appearance was that of an intramural oesophageal haematoma and repeat oesophagoscopy at 10 days showed clear signs of it reducing in size. Follow-up endoscopy at 4 weeks revealed almost complete resolution of the haematoma and the oesophageal mucosa looked normal. She was treated conservatively and her recovery was rapid and complete.

Painful dysphagia, haematemesis and retrosternal chest pain are well documented as common presenting symptoms of spontaneous oesophageal haematoma. ${ }^{5}$ However, the only presenting symptom may be severe retrosternal chest pain. This patient would have fulfilled the criteria for thrombolytic treatment in most institutions where electrocardiographic evidence of myocardial infarction is not essential. The consequences of such 